\title{
LAFFORT Bruno, Les couples mixtes chez les enfants de l'immigration algérienne
}

\section{Gabrielle Varro}

\section{(2) OpenEdition}

10 Journals

Édition électronique

URL : https://journals.openedition.org/remi/4070

DOI : $10.4000 /$ remi. 4070

ISSN : $1777-5418$

Éditeur

Université de Poitiers

\section{Édition imprimée}

Date de publication : 1 décembre 2004

Pagination : 194-196

ISBN : 2-911627-38-5

ISSN : 0765-0752

Référence électronique

Gabrielle Varro, "LAFFORT Bruno, Les couples mixtes chez les enfants de l'immigration algérienne", Revue européenne des migrations internationales [En ligne], vol. 20 - n³ | 2004, mis en ligne le 25 septembre 2008, consulté le 16 avril 2022. URL : http://journals.openedition.org/remi/4070 ; DOI : https://doi.org/10.4000/remi.4070

Ce document a été généré automatiquement le 16 avril 2022.

(c) Université de Poitiers 


\title{
LAFFORT Bruno, Les couples mixtes chez les enfants de l'immigration algérienne
}

\author{
Gabrielle Varro
}

\section{RÉFÉRENCE}

LAFFORT Bruno, Les couples mixtes chez les enfants de l'immigration algérienne, Paris :

L'Harmattan (CREAC « Politique et société »), 2003, 317 p. ISBN : 2-7475-5668-9

1 Contrairement à beaucoup des travaux menés antérieurement sur les couples mixtes, qui ont concerné essentiellement des immigrants ayant épousé un(e) citoyen(ne) du pays d'accueil, le livre de Bruno Laffort porte sur les unions mixtes de la «seconde génération ». Qui est concerné par ce type d'union? Après un rapide "état des lieux » consacré justement aux travaux sur les couples franco-maghrébins de la "première génération ", l'auteur répond à notre curiosité en proposant la définition suivante: "Nous appellerons "couple mixte de la deuxième génération" tout couple formé par une personne née en France de deux parents algériens (ou née en Algérie mais arrivée en France en bas âge) et par une personne née en France de deux parents français » (p. 41). Dans la présentation de son enquête (50 sq.), il précise qu'il s'agit de cinq couples dont la jeune femme est « d'origine algérienne » et de cinq couples dont c'est le jeune homme qui est « d'origine algérienne ».

2 Cette définition et ces précisions ont de quoi surprendre, puisque l'on sait que les enfants d'immigrés sont majoritairement nés en France. Quel critère permet alors de désigner comme couples «mixtes » ceux dont les deux conjoints sont de fait français ? Il s'agit d'évidence d'un choix épistémologique, ou tout au moins interprétatif, de la part du chercheur, consistant à mettre au premier plan de sa conception de l'identité d'un individu ses origines familiales, en n'accordant à sa socialisation dans le pays de naissance et de résidence qu'une valeur secondaire. 
3 On peut ne pas se satisfaire de cette approche, dans le sens que, beaucoup plus que de l'origine (qui peut rester anecdotique dans la biographie des personnes), la mixité d'un couple nous semble dépendre du fait que les conjoints aient été socialisés dans des pays différents. L'une des interviewées le dit d'ailleurs elle-même: "C'est vrai qu'on est de deux origines différentes... mais pas de deux cultures foncièrement différentes, parce que Saïd a grandi en France, il a fait des études à la fac, et puis il ne s'est pas renfermé sur la culture d'origine comme d'autres ont pu le faire " (Séverine, p. 203). En outre, les tableaux (pp. 52 et 58-59) mettent en évidence l'homogamie très générale de ces couples (aux situations socio-professionnelles comparables). Par conséquent, les dire "mixtes » à cause des appartenances de leurs parents, et à l'inverse, employer le terme « endogame » pour un couple «formé par deux personnes d'origine algérienne» (p. 121) - autrement dit, tout analyser en termes des origines familiales des conjoints - peut paraître quelque peu mécanique, faire écran à ce qu'ils peuvent réellement avoir en commun et finir par donner dans le stéréotype ${ }^{1}$.

4 Ces réserves étant faites - non pour censurer car un chercheur est libre de choisir ses définitions mais pour entrer dans le vif du débat - regardons la suite de l'ouvrage. (Disons entre parenthèses qu'on peut regretter les coquilles auxquelles le lecteur s'est habitué dans les ouvrages publiés par L'Harmattan, mais plus sérieusement, l'absence apparente d'une relecture éditoriale attentive, qui aurait permis la suppression d'une certaine redondance dans le plan, voire de certaines appréciations un peu hâtives de l'auteur). B. Laffort a certainement décidé de désigner ces couples comme "mixtes» afin de traduire une réalité sociale incontestable, à savoir non pas tant comment les conjoints se considèrent eux-mêmes mais la manière dont ils sont appréhendés par autrui. De ce point de vue, on peut supposer (et les témoignages que B. Laffort nous donne à entendre soutiennent ce présupposé) que ces couples ne laissent pas l'environnement, les parents et voisins indifférents. Ils sont effectivement perçus comme "mixtes », et d'abord à cause de leurs prénoms (François et Fatima, Saïd et Séverine, Daniel et Nadia, etc.), éventuellement aussi à cause d'autres facteurs qui les "marquent» comme différents voire comme "destinés » à connaître des difficultés plus considérables que celles qui touchent des couples «ordinaires» («non-mixtes»). C'est un point important - sur lequel B. Laffort revient dans sa conclusion - peut-être le plus important, qui justifie à lui seul l'utilisation de l'expression « couple mixte».

5 À travers les entretiens retranscrits ici (à raison d'une trentaine de pages chacun, soit environ 250 pages), nous voyons en effet s'esquisser les auto-portraits des protagonistes, sur lesquels deux ou trois pages de présentation par l'auteur jettent une lumière crue ou tamisée. Dalila: la rupture; Nacera: le mariage musulman en guise de compromis; Léila: patience et longueur de temps; Ali : la rupture, malgré une concession initiale; Daniel : une " prise de distance " partagée; Saïd : l'ouverture et la tolérance; Fatima : la conversion de cœur effectuée par son conjoint. Dans la synthèse qui suit, B. Laffort dégage les thèmes qui lui "semblent les plus caractéristiques de cette "mixité de la deuxième génération" chez les enfants de l'immigration maghrébine, et algérienne en particulier ", dont certains " permettront de réaliser une comparaison avec les couples mixtes formés par les primoarrivants » (p. 255). Parmi ces thèmes, signalons : la rencontre, où apparaît, selon les hommes, la difficulté d'avoir une relation «à l'occidentale » avec une Maghrébine et, selon les femmes, la crainte qu'une relation avec un Maghrébin ne remette «leur émancipation en cause "; une mixité de façade, qui recouvre l'homogamie sociale assez générale des couples «mixtes" constatée dans la plupart des autres enquêtes; la 
relation du couple, où B. Laffort remarque que "les problèmes qui pouvaient affecter les couples mixtes de la première génération au sein même de leur couple, ont totalement disparu " chez eux et que "la solidité de ces unions n'est finalement pas différente de celle de tous les autres couples " (p. 261).

L'ouvrage de B. Laffort vient confirmer les observations d'auteurs qui l'ont précédé dans les recherches sur la mixité conjugale et qui font aujourd'hui partie des connaissances accumulées depuis une trentaine d'années. La question nouvelle qui émerge de son travail sur la « deuxième génération » est de savoir s'il faut continuer à désigner ces couples comme "mixtes». Deux remarques à ce propos: d'une part, B. Laffort semble répondre par l'affirmative en proposant trois hypothèses explicatives, dont la première est que la désignation permet de mesurer l'intégration (réussie) de l'enfant de l'immigration algérienne, bien qu'il ne s'agisse pas vraiment d'« intégration " pour ces Français-es de naissance, comme le font remarquer des interviewé-e-s (p. 284). D'autre part, il répond à cette question épineuse à plusieurs reprises dans son livre, en concluant justement par la différence entre première et deuxième générations. En effet, "on peut dire que les couples mixtes de la première génération ont eu à souffrir à l'intérieur et à l'extérieur de leur union. Les couples [de la deuxième génération...] ne rencontrent plus de tensions internes par rapport à de présupposées différences culturelles mais continuent à souffrir de la pression sociale » (p. 293).

C'est donc plutôt à l'étude de la société française et à ses politiques d'intégration insuffisantes que le livre de Bruno Laffort nous encourage en dernière analyse, afin d'examiner de plus près ce qui peut faire que les couples qu'il a rencontrés, bien que tous deux "français ", suscitent encore l'intérêt pas toujours bienveillant des observateurs. Grâce aux entretiens, qui nous sont rendus in extenso, nous disposons de témoignages qui ont une vraie valeur démonstrative; l'étudiant ou le chercheur en quête de données personnelles concernant le choix du conjoint par des jeunes issus de l'immigration ainsi que par des jeunes de parents déjà français, trouveront ici un «terrain » à exploiter, des situations à analyser, des énoncés à méditer.

\section{NOTES}

1. Le titre de l'ouvrage nous interpelle car dans un couple «mixte» il y a forcément deux conjoints : pourquoi alors s'orienter toujours exclusivement sur le partenaire "marqué »? Pourquoi ne pas titrer par exemple «Les couples mixtes chez des enfants de Français»? La proposition n'est pas absurde, il s'agirait d'inverser pour une fois le point de vue et de voir ce qu'entraîne cette nouvelle catégorisation. 


\section{AUTEURS}

\section{GABRIELLE VARRO}

Laboratoire Printemps, CNRS, UVSQ 